\title{
Optimal Transmit Spectra for Communication in the Presence of Crosstalk and Imperfect Echo Cancellation
}

\author{
Nadeem Ahmed, Richard G. Baraniuk, Donald P. Shaver \\ Department of Electrical and Computer Engineering \\ Rice University, 6100 Main Street \\ Houston, Texas 77005
}

\begin{abstract}
In many communication systems, including Digital Subscriber Lines, performance is severely limited by crosstalk interference. Previous work has presented a general framework for designing optimal transmit spectra for crosstalk avoidance. The technique uses the channel, noise, and interference characteristics to setup and solve an optimization problem which maximizes the capacity of neighboring lines, while maintaining spectral compatibility with other services. This joint signaling and optimal power distribution technique yields significant performance gains over conventional fixed spectra in terms of bit-rates and performance margins. In general, the spectra that result from this scheme have both an echo cancelled and frequency division multiplexed region. To ease the analysis, this technique assumed that the echo canceller has perfect echo rejection capability, which in practice is not true. In this paper, we propose an extension to these techniques, in which we factor the performance of practical echo cancellers into the optimization procedure. When echo rejection is not perfect, as is generally the case, our technique shows significant performance gains over previous techniques. As the performance of the echo canceller increases, our technique converges to the same solution.
\end{abstract}

Keywords - Digital Subscriber Line (DSL), capacity, crosstalk, echo canceller, transmit spectra, power distribution, waterfilling.

\section{INTRODUCTION}

There are many competing broadband techniques for local loop or "last mile service." Digital Subscriber Line (DSL) technology is gaining acceptance as a viable method to meet the continually increasing demand for bandwidth. The various xDSL services eliminate the need for expensive infrastructure upgrades, making it very cost effective broadband access for the huge base of installed telephones based on copper wire.

Telephone lines are packed closely together into binders in a cable. Crosstalk results from the proximity of the lines and significanlty limits achievable bit-rates [1]. The energy of the modulated signals radiate into adjacent copper wires in the same cable binder. The problem is intensified when systems within the same cable binder use the same range of frequencies.

Previous work [2-5] dealing with this problem focused

This work was supported by the National Science Foundation, Texas ATP, and Texas Instruments Leadership University program.

Email: $\{$ nahmed, richb\}@ rice.edu, shaver@ti.com.

Web: http://www.dsp.rice.edu on crosstalk avoidance between same-service lines in a binder, using orthogonal signaling techniques to design optimal transmit spectra. These spectra tell us how to distribute power over frequency. This technique solved an optimization problem which maximized capacity given the channel, noise and crosstalk characteristics. The algorithm produced a transmit spectra, which by design, was spectrally compatible with neighboring services.

In general, the technique of [2-5] produce transmit spectra that have both an echo cancelled region (denoted EQPSD) and a frequency division signaling (FDS) region. In the EQPSD region, both upstream and downstream transmit spectra use the same frequency band, and in the FDS region, upstream and downstream spectra are orthogonal to one another. This technique assumes that in the EQPSD region, after signal processing, residual echo is zero. This implies that echo canceller has infinite echo rejection capability, which in practice is not the case. In many situations the algorithm of [2-5], produce transmit spectra having large echo cancelled regions. The performance of these spectra would be hurt significantly, when used in systems with practical echo cancellers, having finite echo rejection capability. Thus, it is prudent to factor the performance of the echo canceller at hand into the optimization process, to produce a transmit spectra that is optimal for the hardware available.

\section{Definitions And Notation}

Throughout the course of this paper, we will keep our notation consistent with [2-4]. Crosstalk is a significant problem associated with higher frequencies and is typically categorized in one of two forms (Figure 1):

1. Near End Crosstalk (NEXT): interference from neighboring lines arising when signals are transmitted in opposite directions.

2. Far End Crosstalk (FEXT): interference from neighboring lines ariseing when signals are transmitted in the same direction.

Of the two types of crosstalk, NEXT is generally more problematic and causes more interference, as FEXT is 


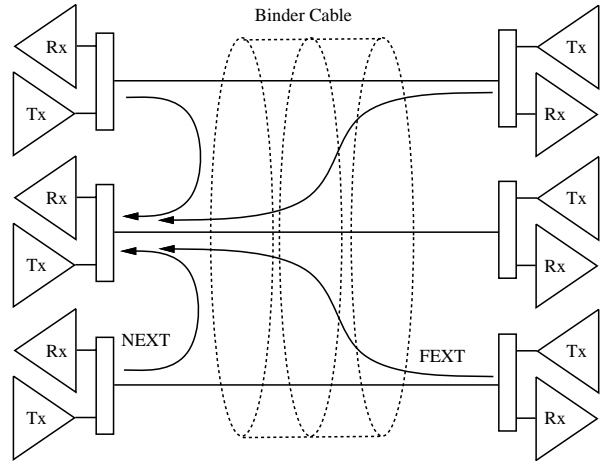

Fig. 1. NEXT and FEXT between neighboring lines in a cable binder

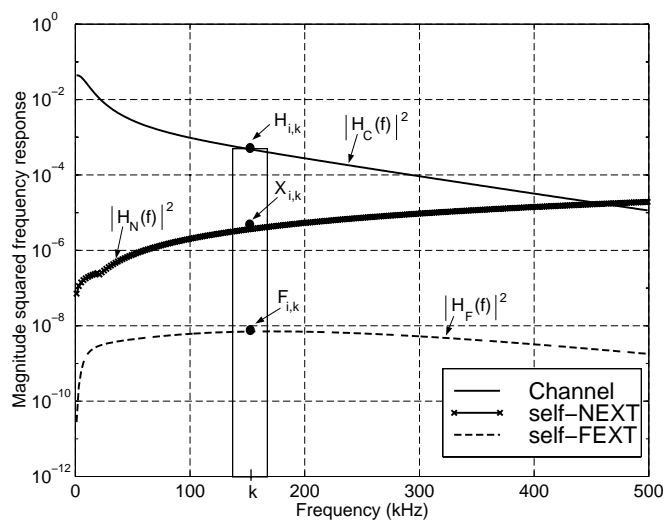

Fig. 2. Magnitude-squared transfer function of the channel (CSA loop 6), 39 self-NEXT interferers, and 39 self-FEXT interferers.

also attenuated as the signal propagates the length of the line. When neighboring lines carry the same service, i.e. HDSL2, the crosstalk is referred to as self-interference, self-NEXT and self-FEXT, respectively. Crosstalk from other services will be referred to as different services (DS) interference, DS-NEXT and DS-FEXT.

Figure 2 illustrates the channel, self-NEXT and self-FEXT transfer functions, denoted $H_{C}(f), H_{N}(f)$, $H_{F}(f)$, respectively. It is assumed that the channel is well characterized by a linear time-invariant system. We divide the total tranmission bandwidth, $B$, into $K$ narrow frequency bins, each of width $W \mathrm{~Hz}$. We choose $K$, such that the channel, self-NEXT and self-FEXT transfer functions are relatively constant in each bin.

We use the following notation for the channel transfer function of line $i$ line in a cable binder [7]

$$
\left|H_{C}(f)\right|^{2}= \begin{cases}H_{i, k} & \text { if }\left|f-f_{k}\right| \leq \frac{W}{2}, \\ 0 & \text { otherwise }\end{cases}
$$

self-NEXT transfer function [11]

$$
\left|H_{N}(f)\right|^{2}= \begin{cases}X_{i, k} & \text { if }\left|f-f_{k}\right| \leq \frac{W}{2} \\ 0 & \text { otherwise }\end{cases}
$$

and self-FEXT transfer function [11]

$$
\left|H_{F}(f)\right|^{2}= \begin{cases}F_{i, k} & \text { if }\left|f-f_{k}\right| \leq \frac{W}{2} \\ 0 & \text { otherwise. }\end{cases}
$$

where $f_{k}$ are the center frequency of the $K$ bins with index $k \in\{1, \ldots, K\}$. As we consider only real signals with symmetric frequency responses, we only consider quantitites over the non-negative frequency region.

We will be looking at the effect of imperfect echo cancellers, so let us denote

$$
|E(f)|^{2}= \begin{cases}E_{i, k} & \text { if }\left|f-f_{k}\right| \leq \frac{W}{2} \\ 0 & \text { otherwise. }\end{cases}
$$

the residual echo (after echo cancellation) as a fraction of total power in bin $k$. The residual echo in that bin would then be $E_{k} P_{k}$, where $P_{k}$ is the power placed in bin $k$.

Interference from other services, DS-NEXT and DSFEXT, is denoted $D S_{N}(f)$ and $D S_{F}(f)$. Additive gaussian noise (AGN), denoted $N_{o}(f)$, can be grouped with DS-NEXT and DS-FEXT to form a total noise term

$$
N(f):=N_{o}(f)+D S_{N}(f)+D S_{F}(f) .
$$

Upstream and downstream transmit power spectral densities (PSDs) are denoted $S^{u}(f)$ and $S^{d}(f)$, while $s^{u}(f)$ and $s^{d}(f)$ denote the PSDs in bin $k$.

\section{OPTIMAL TRANSMIT SPECTRA}

Generally, self-FEXT is far less troublesome than selfNEXT (Figure 2), as FEXT is also attenuated as it travels the length of the line (Figure 1). We assume that selfNEXT dominates self-FEXT and as such, the crosstalk avoidance technique described in this paper and in [3-5] focuses on self-NEXT rejection, with self-FEXT factoring into the optimization process.

The goal is to maximize the capacity of users of a given service (i.e. all HDSL2 users in a cable binder), while minimizing the performance degradation of other services. As previously mentioned, NEXT is the dominant interference in XDSL service. A simple way to eliminate self-NEXT is to use an orthogonal signaling technique. It has been shown in [3] that under a power constraint, FDS is the optimal orthogonal signaling technique. To eliminate self-NEXT using FDS, we can force upstream and downstream transmitters to use disjoint frequency bands. The upstream and downstream transmissions are orthogonal and can easily be separated by their respective receivers. Moving to an FDS scheme reduces the bandwidth available to each transmitter by $1 / 2$ of the original. There is a tradeoff as FDS eliminates self-NEXT, increasing capacity, while also reducing the bandwidth available and decreasing capacity. Thus, when self-NEXT is not high enough to warrant FDS, both upstream and downstream transmitters should have the same spectrum, or EQPSD signaling.

For the purpose of this paper, we consider symmetric services (i.e. HDSL2) where the upstream capacity, $C^{u}$, 
and downstream capacity, $C^{d}$ are equal. With slight modifications, this technique can also be applied to asymmetric services, including ADSL. Our optimization concentrates on maximizing upstream and downstream capacity, given a total power constraint, $P_{\text {tot }}$, and the equal capacity constraint $C^{u}=C^{d}$.

To solve this problem, one would generally divide the channel into a number of frequency bins, and maximize the sum of the capacity in each bin subject to the constraints. To further complicate matters, within each bin, the optimiziation routine must also decide which signaling scheme to choose, EQPSD or FDS. More specifically, the solution to the optimization problem becomes

$$
C=\max _{k=1,2, \ldots, K} \sum_{k=1}^{K} C_{k}\left(P_{k}, H_{k}, X_{k}, F_{k}, E_{k}, N_{k}\right)
$$

subject to the constraints $0 \leq P_{k} \leq P_{\text {tot }}$ and $\sum_{k=1}^{K} P_{k} \leq$ $P_{\text {tot }}$. Each $C_{k}$ is the greater of the capacities achieved by either using EQPSD or FDS signaling in that particular bin. The number of bins is generally large; to make the channel, NEXT and FEXT transfer functions appear flat. Therefore, the problem becomes prohibitively computationally complex.

The problem can greatly be simplified when the channel and crosstalk transfer functions are monotonic functions of frequency [2-4]. When this occurs, as is generally the case in the xDSL environment, we get an optimal spectra that only has EQPSD signaling to up to a crossover frequency, and FDS after. The problem can be broken into two parts, determining the EQPSD to FDS crossover point, and power distribution within each region.

\section{A. Decision Regions}

We begin our analysis by first deciding which signaling scheme to use within each bin. We divide each bin in half and use the symmetric signaling scheme

$$
\begin{aligned}
& s^{u}(f)= \begin{cases}\alpha \frac{2 P_{k}}{W} & \text { if } 0 \leq f \leq \frac{W}{2}, \\
(1-\alpha) \frac{2 P_{k}}{W} & \text { if } \frac{W}{2}<f \leq W, \\
0 & \text { otherwise, }\end{cases} \\
& s^{d}(f)= \begin{cases}(1-\alpha) \frac{2 P_{k}}{W} & \text { if } 0 \leq f \leq \frac{W}{2}, \\
\alpha \frac{2 P_{k}}{W} & \text { if } \frac{W}{2}<f \leq W, \\
0 & \text { otherwise. }\end{cases}
\end{aligned}
$$

where $P_{k}$ is the average power over the bandwidth $W$ in bin $k$ and $0.5 \leq \alpha \leq 1$. The parameter $\alpha$ controls the distribution of power within the bin. When $\alpha=0.5$, the power distribution in both the upstream and downstream directions are equal, hence EQPSD signaling. At
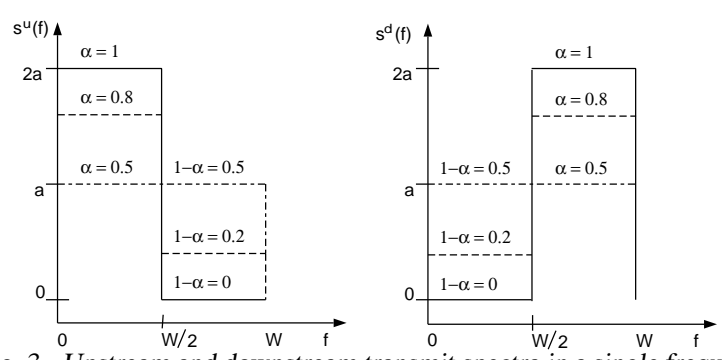

Fig. 3. Upstream and downstream transmit spectra in a single frequency bin ( $\alpha=0.5 \Rightarrow$ EQPSD signaling and $\alpha=1 \Rightarrow$ FDS signaling).

the other extreme, when $\alpha=1$, upstream and downstream bands are completely disjoint, hence FDS signaling. These extremes, along with other possible transmit spectra, are illustrated in Figure 3.

For ease of notation, in this derivation we set

$$
H:=H_{i, k}, \quad X:=X_{i, k}, \quad F:=F_{i, k}, \quad E:=E_{i, k}
$$

As in [3], we define acheivable rate as

$$
\begin{array}{r}
R_{A}\left(s^{u}(f), s^{d}(f)\right)=\int_{0}^{W} \log _{2}[1+ \\
\left.\frac{s^{u}(f) H}{N+s^{d}(f)(X+E)+s^{u}(f) F}\right] d f,
\end{array}
$$

then

$$
\begin{gathered}
c^{u}=\max _{0.5 \leq \alpha \leq 1} R_{A}\left(s^{u}(f), s^{d}(f)\right) \quad \text { and } \\
c^{d}=\max _{0.5 \leq \alpha \leq 1} R_{A}\left(s^{d}(f), s^{u}(f)\right) .
\end{gathered}
$$

Since the upstream and downstream spectra are symmetric, the channel capacities $c^{u}$ and $c^{d}$ will always be equal. Thus, it suffices to maximize only one of these terms. We will only consider upstream capacity, $c^{u}$, and introduce the notation $R_{A}$ for $R_{A}\left(s^{u}(f), s^{d}(f)\right)$. Substituting equations (7) and (8) into (11), we obtain the following expression for upstream capacity

$$
\begin{aligned}
c^{u} & =\max _{0.5 \leq \alpha \leq 1} \frac{W}{2 \ln (2)} \\
& \left\{\ln \left[1+\frac{\alpha G H}{1+(1-\alpha) G(X+E)+\alpha G F}\right]\right. \\
& \left.+\ln \left[1+\frac{(1-\alpha) G H}{1+\alpha G(X+E)+(1-\alpha) G F}\right]\right\}(1)
\end{aligned}
$$

where $G=\frac{2 P_{k}}{N W}$. By maximizing this expression, we obtain $R_{A}$. Taking the derivative with respect to $\alpha$, and after some simplification, we obtain

$$
\begin{array}{r}
\frac{\partial R_{A}}{\partial \alpha}=G(2 \alpha-1)[2([X+E]-F)+ \\
\left.G\left([X+E]^{2}-F^{2}\right)-H(1+G F)\right] L
\end{array}
$$


where $L>0$. If we set the derivative to zero, we see that there is a single stationary point at $\alpha=0.5$. The achievable data rate is monotonic for $\alpha \in(0.5,1]$. As a result we realize that if $\alpha=0.5$ maximizes $R_{A}$, then EQPSD signaling is optimal in this bin. If $\alpha=0.5$ minimizes $R_{A}$, then $\alpha=1$ maximizes it (from the monotonicity of $R_{A}$ ), meaning FDS is optimal in this bin. Thus we see that for each bin, either EQPSD or FDS signaling is optimal. Test conditions for signaling in a particular bin are obtained by solving (13). They result in:

$$
\begin{gathered}
G=\frac{2 P_{k}}{N W} \underset{\text { FDS }}{\gtrless} \frac{H-2(X+E-F)}{(X+E)^{2}-F^{2}-H F} \\
\text { if }(X+E)^{2}-F^{2}-H F<0 \text { and } \\
G=\frac{2 P_{k}}{N W} \underset{\text { EQPSD }}{\stackrel{\text { FDS }}{<} \frac{H-2(X+E-F)}{(X+E)^{2}-F^{2}-H F}}
\end{gathered}
$$

if $(X+E)^{2}-F^{2}-H F>0$

We find that all bins to the left of crossover bin $M_{E}$ use EQPSD signaling, while all bins to the right of crossover bin $M_{F}$ use FDS signaling. The signaling schemes for the bins in the region $\left(M_{E}, M_{F}\right)$ cannot be determined with these test conditions, and the true optimal crossover point form the EQPSD to FDS signaling, $M_{E 2 F}$, lies somewhere in between.

\section{B. Power Distribution}

We first look at the distribution of power in the EQPSD region. Since both upstream and downstream spectra are using the same frequency band in the region, echo, NEXT and FEXT along with Gaussian noise limit our performance. To determine the optimal distribution of power over frequency, we begin with the formula for parallel independent Gaussian channels.

$$
C=\sum_{k=1}^{M_{E 2 F}} \frac{B}{2 K} \log _{2}\left(1+\frac{P_{k} H_{k}}{N_{k}+P_{K}\left(F_{k}+X_{k}+E_{k}\right)}\right)
$$

with the power constraint $\sum_{k=1}^{M_{E 2 F}} P_{k}=P_{E}$. The optimal power distribution is obtained by following a modified waterfilling solution similar to that of [10]. Complete details can be found in [6].

Waterfilling in the FDS region is done in a similar manner. However, since upstream and downstream spectra are using disjoint frequency bands, self-NEXT and echo are completely eliminated. Therefore, we begin with the following equation for parallel Gaussian channels in the FDS

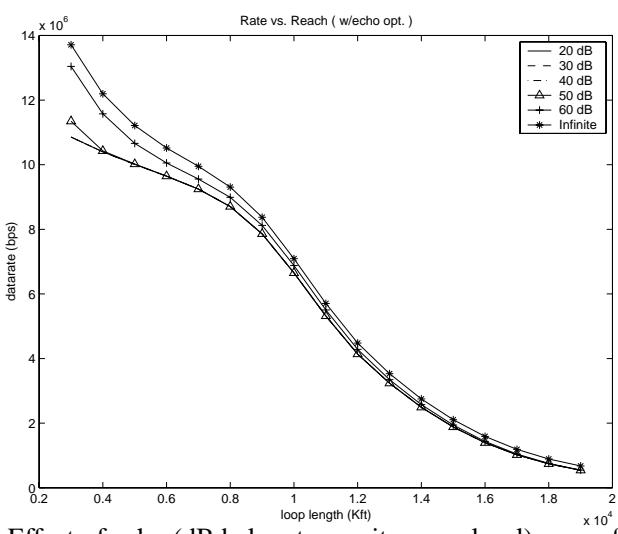

Fig. 4. Effect of echo (dB below transmit power level) on performance of algorithm factoring echo into the optimization process.

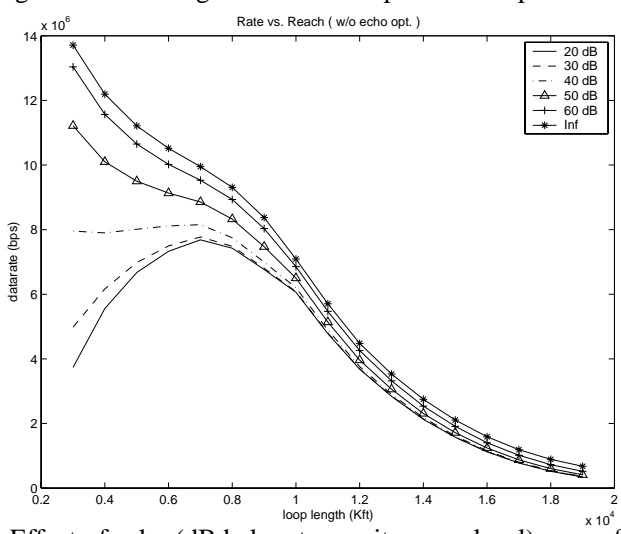

Fig. 5. Effect of echo ( $\mathrm{dB}$ below transmit power level) on performance of algorithm not factoring echo into the optimization process.

region

$$
C=\sum_{k=M_{E 2 F}+1}^{K} \frac{W}{2 K} \log _{2}\left(1+\frac{P_{k} H_{k}}{N_{k}+P_{k} F_{k}}\right)
$$

with the power constraint $\sum_{k=M_{E 2 F}+1}^{K} P_{k}=P_{F}$ and follow in a manner similar to above.

\section{Simulation Results}

The environment for the simulation has each HDSL2 modem having 10 other self-interferors (self-NEXT and self-FEXT) and 10 ISDN interferors (DS-NEXT and DSFEXT) in the cable binder. Data rate is calculated based on a $6 \mathrm{~dB}$ performance margin, with no coding gain. Figure 4 illustrates the performance of our algorithm, in terms of total data rate (sum of upstream and downstream capacity) versus loop length (distance between upstream and downstream modems). Several curves are shown, each for a different level of residual echo (indicated in $\mathrm{dB}$ below transmit power level). Figure 5 illustrates the same information but for the algorithm in [2-5]. Figure 6 indicates, $M_{E 2 F}$, the EQPSD to FDS crossover bin for our algorithm under various levels of residual echo, and for [2-5] (which doesn't depend on echo level). 


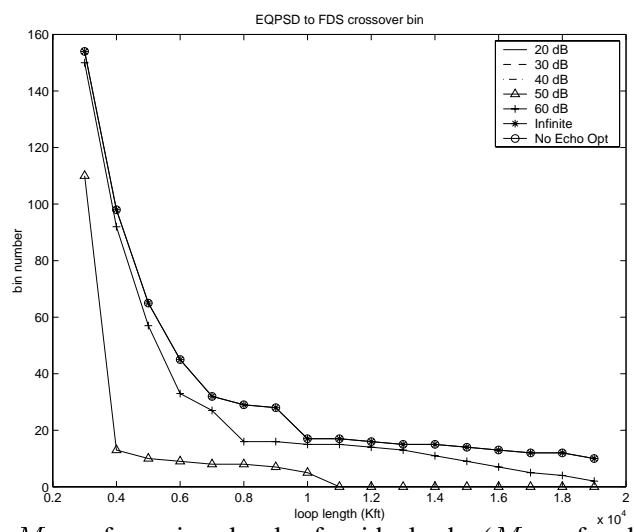

Fig. 6. $M_{E 2 F}$ for various levels of residual echo $\left(M_{E 2 F}\right.$ for algorithm not considering echo included for reference)

\section{Discussion AND CONClusions}

By looking at the curves in Figure 4 and comparing them to the corresponding curves in Figure 5, we see that our optimization scheme significantly outperforms the one developed in [2-5], when residual echo is present.

Both algorithms choose between EQPSD and FDS signaling, to reject self-NEXT, in a fashion that maximizes overall data rate. From equation (11) we see that residual echo acts similarly to self-NEXT. Consider an environment with low self-NEXT but high residual echo. The algorithm in [2-5] sees only low self-NEXT and incorrectly chooses EQPSD signaling. In the same situation, our algorithm also sees the high echo, and chooses FDS.

Figure 6 shows the EQPSD to FDS crossover point for our algorithm. The crossover point for [2-5] is also indicated. Clearly our algorithm uses EQPSD more sparingly, as it considers the effect of residual echo, which is only present in the echo cancelled region. Generally, we have seen that the shorter the loop length, the smaller the effect of self-NEXT. Since there is less need for FDS, we have larger EQPSD regions. Figure 6 shows this trend, as shorter loops lengths have $M_{E 2 F}$ occur at higher frequencies. This helps us understand why the difference in performance between the two algorithms is greater for shorter loops lengths.

We also notice that when the residual echo becomes increasingly small, both techniques converge to the same spectra, seen by comparing Figures 4 and 5. The spectra that our algorithm converges to is the one found in [2-5]. As residual echo approaches zero it does not impact the performance of the EQPSD region and we are left with the spectra from [2-5].

When residual echo is large, the optimal spectra consists solely of an FDS region (Figure 6), and as the echo becomes smaller we start seeing an EQPSD region. More specifically, when the magnitude of the echo, becomes roughly the same as that of the self-NEXT transfer function, we begin to see an EQPSD region. When it is larger than this, it swamps out the self-NEXT and forces the sys- tem to choose an FDS solution.

This gives us some insight into system design. When echo canceller performance is poor enough to swamp out self-NEXT, we almost entirely see an FDS solution. It would be cost effective not to include the extra hardware on a modem for echo cancellation capability, we would not see any performance gain (no EQPSD region). Here the optimal solution, simply the modified waterfilling solution described in section III-B, gives us significantly better performance than the combined EQPSD and FDS solution predicted by [2-5]. When echo rejection is moderate (i.e. the residual echo is roughly the same order of magnitude as self-NEXT), then we see a solution that has both EQPSD and FDS. This solution has significantly better performance than [2-5], especially at shorter loops lengths, as it uses EQPSD more sparingly. With excellent echo canceller performance, our solution converges to the optimal solution (in the presence of no echo), predicted in [2-5].

\section{REFERENCES}

[1] I. Kalet, “The Multitone Channel," IEEE Trans. Commun., vol. 37, no. 2, Feb. 1989.

[2] A. Sendonaris, V. Veeravalli and B. Aazhang, "Joint Signaling Strategies for Approaching the Capacity of Twisted Pair Channels," IEEE Trans. Commun., vol. 46, no. 5, May 1998.

[3] R.V. Gaikwad and R.G. Baraniuk, "Spectral Optimization and Joint Signaling Techniques for Communication in the Presence of Crosstalk," Technical report \#9806, ECE Department, Rice University, July 1998. Web: www.dsp.rice.edu/rohitg/Papers/tr9806.ps

[4] R.V. Gaikwad and R.G. Baraniuk, "Optimal Signialing Strategies for Symmetric and Asymmetric Bit-Rate Communication Services in the Presence of Crosstalk," Technical report \#9901, ECE Department, Rice University, January 1999. Web: www.dsp.rice.edu/rohitg/Papers/tr9901.ps

[5] R.V. Gaikwad and R.G. Baraniuk, "Optimal Transmit Spectra for HDSL2," ANSI T1E1 contribution T1E1.4/98-162R1.

[6] N. Ahmed, R.G. Baraniuk, D.P. Shaver, "Optimal Transmit Spectra for Communication in the Presence of Crosstalk and Imperfect Echo Cancellation," In Preparation.

[7] American National Standard for Telecommunications, "Network and Customer Installation Interfaces-Asymmetric Digital Subscriber Line (ADSL) Metallic Interface," T1.413-1995.

[8] R.G. Gallager, "Information Theory and Reliable Communication," New York: Wiley, 1968.

[9] T.M. Cover and J.A. Thomas, "Elements of Information Theory," New York: Wiley, 1991.

[10] J.T. Aslanis and J.M. Cioffi, "Achievable Information Rates on Digital Subscriber Loops: Limiting Information Rates with Crosstalk Noise," IEEE Trans. Commun., vol. 40, no. 2, Feb. 1992.

[11] K.J. Kerpez, "Full-duplex 2B1Q Single-pair HDSL Performance and Spectral Compatibility," T1E1 contribution T1E1.4/95-127.

[12] K.J. Kerpez, "Near-End Crosstalk is almost Gaussian," IEEE Trans. Commun., vol. 41, no. 1, Jan. 1993. 